\title{
Hydrodynamic Drag During Gliding in Swimming
}

\author{
Daniel A. Marinho, ${ }^{1,2,3}$ Victor M. Reis, ${ }^{2,3}$ Francisco B. Alves, ${ }^{4}$ João P. Vilas-Boas, ${ }^{5}$ \\ Leandro Machado, ${ }^{5}$ António J. Silva, ${ }^{2,3}$ and Abel I. Rouboa ${ }^{2,6}$ \\ 1 University of Beira Interior; ${ }^{2}$ University of Trás-os-Montes and Alto Douro; \\ ${ }^{3}$ Centre for Research in Sports, Health, and Human Development (Vila Real, Portugal); \\ ${ }^{4}$ Technical University of Lisbon; ${ }^{5}$ University of Porto; 6 University of Pennsylvania
}

\begin{abstract}
This study used a computational fluid dynamics methodology to analyze the effect of body position on the drag coefficient during submerged gliding in swimming. The k-epsilon turbulent model implemented in the commercial code Fluent and applied to the flow around a three-dimensional model of a male adult swimmer was used. Two common gliding positions were investigated: a ventral position with the arms extended at the front, and a ventral position with the arms placed along side the trunk. The simulations were applied to flow velocities of between 1.6 and $2.0 \mathrm{~m} \cdot \mathrm{s}^{-1}$, which are typical of elite swimmers when gliding underwater at the start and in the turns. The gliding position with the arms extended at the front produced lower drag coefficients than with the arms placed along the trunk. We therefore recommend that swimmers adopt the arms in front position rather than the arms beside the trunk position during the underwater gliding.
\end{abstract}

Marinho is with the University of Beira Interior, Covilhã, Portugal; the University of Trás-os-Montes and Alto Douro, Vila Real, Portugal; and the Centre of Research in Sports, Health and Human Development, Vila Real, Portugal. Reis is with the University of Trás-os-Montes and Alto Douro, Vila Real, Portugal, and the Centre of Research in Sports, Health and Human Development, Vila Real, Portugal. Alves is with the Faculty of Human Kinetics, Technical University of Lisbon, Lisbon, Portugal. Vilas-Boas is with the Faculty of Sport, University of Porto, Porto, Portugal. Machado is with the Faculty of Sport, University of Porto, Porto, Portugal. Silva is with the University of Trás-os-Montes and Alto Douro, Vila Real, Portugal, and the Centre of Research in Sports, Health and Human Development, Vila Real, Portugal. Rouboa is with the University of Trás-os-Montes and Alto Douro, Vila Real, Portugal, and the Department of Mechanical Engineering and Applied Mechanics, University of Pennsylvania, Philadelphia, PA.
Keywords: human body, numerical simulations, computational fluid dynamics, forces

The underwater phases of swimming after starts and turns are a large and important component of the total event time in modern swimming. Accordingly, Guimarães \& Hay (1985) submit, for instance, that it is essential to minimize the hydrodynamic drag during the gliding. Thus, the swimmer must adopt the most hydrodynamic position possible. Race analysis has suggested that rather than the start position used by the swimmer it is his body alignment under the water that mostly determines the success of the start (Vilas-Boas et al., 2000; Cossor \& Mason, 2001).

The passive drag of swimmers moving underwater in a streamlined position has been measured experimentally (for example, Jiskoot \& Clarys, 1975; Lyttle et al., 2000). These studies revealed the difficulties involved in conducting such experimental research. An alternative approach is to apply the numerical technique of computational fluid dynamics to determine a swimmer's passive drag.

The first application of computational fluid dynamics to swimming was conducted by Bixler \& Schloder (1996). They used a two-dimensional numerical analysis to evaluate the effects of accelerating a hand-sized disc through the water. Additional research using computational fluid dynamics techniques was performed by Rouboa et al. (2006) to evaluate the steady and unsteady propulsive force of a swimmer's hand and arm. Their results suggested that a three-dimensional computational fluid dynamics analysis of a human form could provide useful information about swimming. This was already confirmed by Alves et al. (2007), in the upper arm propulsion, and by Bixler et al. (2007), in the analysis of an entire swimmer's body drag. Hence, the main aim of this study was to analyze the effect in the drag coefficient of the use of two distinct ventral positions during the underwater gliding in swimming, applying 
computational fluid dynamics. A second aim was to study the relative contributions of the skin friction drag and the pressure drag for the total drag during the gliding.

\section{Methods}

\section{Three-Dimensional Model}

To obtain the geometry of a human body, a model was created in CAD (computer-aided design), based on the anthropometrical characteristics of a group of elite national-level male swimmers. The surfaces of the swimmer were then developed using Gambit, a geometry modeling program of Fluent (Fluent, Inc. Hannover, USA), which provides sophisticated computational fluid dynamics software. These surfaces were then meshed, creating the volume mesh that was imported into the Fluent computational fluid dynamics program for analysis (Figure 1).

\section{Computational Fluid Dynamics Model}

The swimmer was modeled as if he were gliding underwater in one of two distinct ventral positions. The first position was a streamlined position, with the arms extended at the front. This is the shape usually adopted after the start and after pushing off from the wall after a turn. The second position was with the arms along the trunk. This is the shape adopted by the swimmers during the second gliding phase after a turn in breaststroke.

The computational fluid dynamics analyses were performed with the body in a horizontal position with an attack angle of $0^{\circ}$. The attack angle was defined as the angle between a horizontal line and a line drawn from the vertex to the ankle bone.

The swimmer's model used for the analysis was $1.87 \mathrm{~m}$ tall with head, chest, waist, and hip circumferences of $0.57 \mathrm{~m}, 1.04 \mathrm{~m}, 0.85 \mathrm{~m}$, and $0.95 \mathrm{~m}$, respec- tively. In the streamlined position, the model had a finger-to-toe length of $2.37 \mathrm{~m}$ and in the position with the arms along the trunk the distance from vertex to toe was $1.92 \mathrm{~m}$.

The boundary conditions of the computational fluid dynamics model were designed to represent the geometry and flow conditions of a part of a lane in a swimming pool. The water depth of the model was $1.80 \mathrm{~m}$ with a $2.50 \mathrm{~m}$ width. The length was $8.0 \mathrm{~m}$ in the streamlined position and $7.55 \mathrm{~m}$ in the position with the arms along the trunk, allowing in both situations the same flow conditions behind and in front of the swimmer. In both positions, the distance to the front surface was $2.0 \mathrm{~m}$ and to the back surface was $3.63 \mathrm{~m}$. The swimmer model middle line was placed at a water depth of $0.90 \mathrm{~m}$, equidistant from the top and bottom surfaces (Figure 2).

The model's body surface had roughness parameters of zero. The whole domain was meshed with 900 million cells. The grid was a hybrid mesh composed of prisms and pyramids. Significant efforts were conducted to ensure that the model would provide accurate results, namely by decreasing the grid node separation in areas of high velocity and pressure gradients.

Steady-state computational fluid dynamics analyses were performed using the Fluent code, and the drag coefficient was calculated for velocities ranging from 1.60 to $2.0 \mathrm{~m} \cdot \mathrm{s}^{-1}$ in increments of $0.10 \mathrm{~m} \cdot \mathrm{s}^{-1}$. Flow velocities were chosen to be within the range of typical underwater gliding velocities at the start and in the turns. The Fluent code solves flow problems by replacing the Navier-Stokes equations with discretized algebraic expressions that can be solved by iterative computerized calculations. Fluent uses the finite volume approach, where the equations are integrated over each control volume.

We used the segregated solver with the standard $\mathrm{k}$-epsilon turbulence model because this turbulence model was shown to be accurate with measured values in a previous research (Moreira et al., 2006).

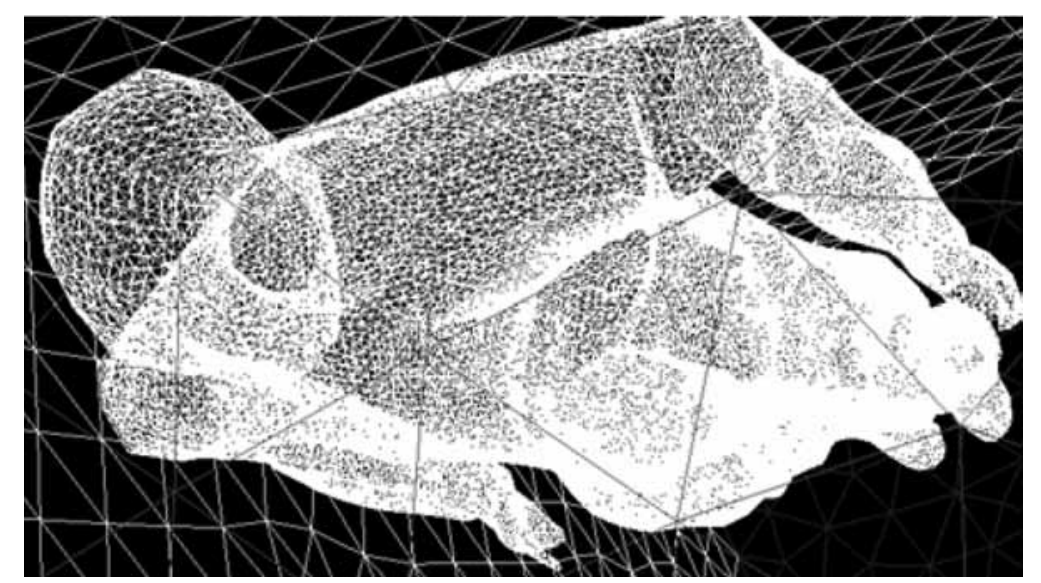

Figure 1 - Swimmer's model geometry with the surfaces meshed using Gambit. An example for the position with the arms along the trunk is presented. 


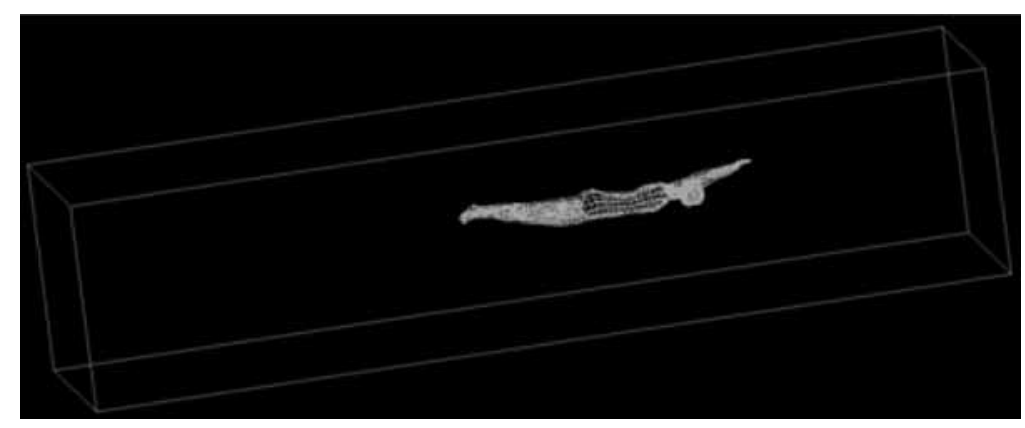

Figure 2 - Computational fluid dynamics model geometry with the swimmer with the arms extended at the front. The water depth is $1.80 \mathrm{~m}$, the width is $2.50 \mathrm{~m}$, and the length is $8.0 \mathrm{~m}$.

Table 1 Drag coefficient values and the contribution of pressure and skin friction drag for the total drag to each velocity and for the two different gliding positions

\begin{tabular}{|c|c|c|c|c|c|c|c|c|c|c|}
\hline \multirow{3}{*}{$\begin{array}{r}\begin{array}{r}\text { Velocity } \\
\left(\mathrm{m} \cdot \mathbf{s}^{-1}\right)\end{array} \\
1.6\end{array}$} & \multicolumn{5}{|c|}{ Drag Coefficient (Arms Along the Trunk) } & \multicolumn{5}{|c|}{ Drag Coefficient (Arms Extended at the Front) } \\
\hline & \multirow{2}{*}{$\begin{array}{c}\text { Total drag } \\
0.824\end{array}$} & \multicolumn{2}{|c|}{ Pressure drag } & \multicolumn{2}{|c|}{ Friction drag } & \multirow{2}{*}{$\begin{array}{c}\text { Total drag } \\
0.480\end{array}$} & \multicolumn{2}{|c|}{ Pressure drag } & \multicolumn{2}{|c|}{ Friction drag } \\
\hline & & 0.758 & $91.98 \%$ & 0.066 & $8.02 \%$ & & 0.417 & $86.95 \%$ & 0.063 & $13.05 \%$ \\
\hline 1.7 & 0.782 & 0.719 & $91.99 \%$ & 0.063 & $8.01 \%$ & 0.475 & 0.413 & $86.98 \%$ & 0.062 & $13.02 \%$ \\
\hline 1.8 & 0.763 & 0.702 & $92.01 \%$ & 0.061 & $7.99 \%$ & 0.432 & 0.376 & $87.01 \%$ & 0.056 & $12.99 \%$ \\
\hline 1.9 & 0.762 & 0.701 & $92.04 \%$ & 0.061 & $7.96 \%$ & 0.431 & 0.375 & $87.03 \%$ & 0.056 & $12.97 \%$ \\
\hline 2.0 & 0.736 & 0.677 & $92.05 \%$ & 0.059 & $7.95 \%$ & 0.428 & 0.373 & $87.04 \%$ & 0.055 & $12.96 \%$ \\
\hline
\end{tabular}

All numerical computational schemes were second order (Rouboa \& Silva, 2007), which provides a more accurate solution than first-order schemes. We used a turbulence intensity of $1.0 \%$ and a turbulence scale of $0.10 \mathrm{~m}$. The water temperature was $28^{\circ} \mathrm{C}$ with a density of $998.2 \mathrm{~kg} \cdot \mathrm{m}^{-3}$ and a viscosity of $0.001 \mathrm{~kg} \cdot(\mathrm{m} \cdot \mathrm{s})^{-1}$. Incompressible flow was assumed.

In human swimming, the total drag is composed of the skin friction drag, pressure drag, and wave drag. Skin friction drag is attributed to the forces tending to slow the water flowing along the surface of a swimmer's body. It depends on the velocity of the flow, the surface area of the body, and the characteristics of the surface. Pressure drag is caused by the pressure differential between the front and the rear of the swimmer and it is proportional to the square of swimming velocity, the density of water, and the cross-sectional area of the swimmer. Finally, swimming at the water surface is constrained by the formation of surface waves leading to wave drag. In this study, we considered hydrodynamic drag depending only on the skin friction and pressure drag since the model was placed $0.90 \mathrm{~m}$ underwater. These two drag components were computed by Fluent software.

\section{Statistical Analyses}

To analyze the relationship between the velocity and the drag coefficient, regression lines between these parameters were computed. The regression equations were calculated and the $R^{2}$ value was used as a measure of the robustness of the model.

\section{Results}

Table 1 shows the drag coefficient values produced by both models: with the arms along the trunk and with the arms extended at the front. The percentage and the absolute values of total drag resulting from skin friction and pressure drag are also presented. For all the velocities, the drag coefficient of the position with the arms extended at the front was lower than the drag coefficient of the position with the arms along the trunk. Moreover, the pressure drag was dominant, with a percentage of about $92 \%$ and $87 \%$ of the total drag, in the position with the arms along the trunk and with the arms extended at the front, respectively. The absolute values of skin friction drag were quite similar in both positions.

On the other hand, in both positions, the drag coefficient of the model decreased with the velocity (Table 1 and Figure 3).

\section{Discussion}

The main aim of this study was to analyze the drag coefficient arising from the use of two different gliding positions in swimming, through computational fluid dynamics.

The drag coefficient changed slightly from 0.824 at $1.60 \mathrm{~m} \cdot \mathrm{s}^{-1}$ to 0.736 at $2.00 \mathrm{~m} \cdot \mathrm{s}^{-1}$, in the position with the arms along the trunk, and from 0.480 at $1.60 \mathrm{~m} \cdot \mathrm{s}^{-1}$ to 0.428 at $2.00 \mathrm{~m} \cdot \mathrm{s}^{-1}$, in the position with the arms extended at the front. The inverse relationship between the drag coefficient and the velocity found in the current 


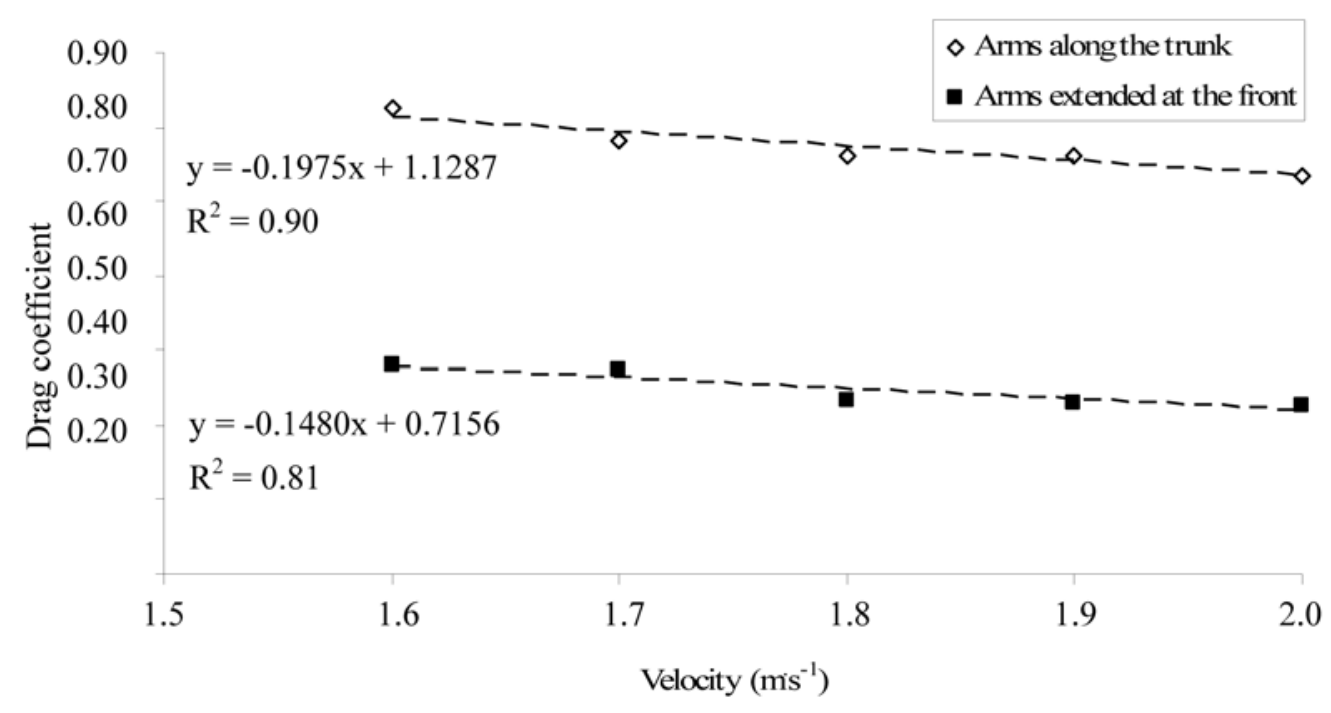

Figure 3 - Relationship between the drag coefficient and the velocity for the two different gliding positions. The regression equations and the $R^{2}$ values are also presented.

study seems to correspond to what happens in experimental situations with the human body totally submersed (Jiskoot \& Clarys, 1975; Lyttle et al., 2000).

Moreover, the gliding position with the arms extended at the front presented lower drag coefficient values than the position with the arms placed along the trunk. This body position, with the arms at the front, is mostly accepted by the swimming technical and scientific communities as the most hydrodynamic one, being called the streamlined position (Guimarães \& Hay, 1985). The position with the arms extended at the front seems to be the one that allows a higher reduction of the negative hydrodynamic effects of the human body morphology: a body with various pressure points due to the large changes in its shape. This position seems to smooth the anatomical shape especially at the head and shoulders. Considering the breaststroke turn, the first gliding, performed with the arms at the front, must be emphasized in relation to the second gliding, performed with the arms along the trunk.

Bixler et al. (2007) and Silva et al. (2008) demonstrated the validity of computational fluid dynamics analysis as a tool to examine the water flow around a submerged swimmer's body. This form of research has opened a new gate of analysis into the swimming hydrodynamics and has been shown to hold promise as a way to assess the flow characteristics and associated drag forces experienced by swimmers, for instance, in different gliding positions after starts and turns.

Another aim of the current study was to analyze the influence of the skin friction drag and the pressure drag in the total drag during the gliding. We choose a pool depth of $1.80 \mathrm{~m}$, with the swimmer model placed at the midpoint between top and bottom, to avoid significant wave drag, limiting our research to the influence of the pressure drag and the skin friction drag in the total drag coefficient. Lyttle et al. (1999) concluded that there is no significant wave drag when a typical adult swimmer is at least $0.6 \mathrm{~m}$ under the water's surface.

The computed drag forces components showed that for both gliding positions the pressure drag was dominant. Nevertheless, skin friction drag was by no means negligible, presenting an absolute value of about 0.06 . This drag component represented $\approx 13 \%$ and $\approx 8 \%$ of total drag in the position with the arms extended at the front and with the arms along the trunk, respectively. However, these values are based on the swimmer model's surface having a zero roughness. Therefore, the development of roughness parameters for human skin would allow a more accurate computational fluid dynamics model to be built in further studies. Since this task is still in development, we assumed to conduct our simulations based upon the swimmer's surface having a zero roughness. We chose this value as a first step in the application of numerical simulation techniques in swimming research, using a three-dimensional model of a whole human body. We had to opt between a certain value and a zero value. Indeed, we simulated a situation as the swimmer was shaved (smoothed), with roughness zero. In our opinion, the change in the roughness parameter would affect each body position in approximately the same way and our main finding would be the same. However, the contribution of each drag component would possibly be a little different with the use of a roughness skin value. Nevertheless, we are convinced that the pressure drag would be dominant and the skin friction drag would be important as well. How this relative contribution would be changed is a very interesting question, which could lead to further research. But one can speculate about this. On one hand, if the surface roughness were increased in the model, the skin friction drag would probably be higher. It is expected that the 
surface roughness increase could lead to increase the turbulence around the surface, thus increasing skin friction drag. On the other hand, if the surface roughness were increased the pressure drag could be reduced. The boundary layer, which would be mainly laminar, would change into a turbulent one (Massey, 1989). When the flow regimen is laminar, separation at the body surface starts almost as soon as the pressure gradient becomes adverse and a larger wake forms, whereas when the flow regimen is turbulent, separation is delayed and the corresponding wake is smaller, thus decreasing pressure drag. The importance of keeping the boundary layer attached to the swimmer body surface is so important that swimwear manufacturers sometimes purposely cause the boundary layer to become turbulent (Polidori et al., 2006).

Moreover, since the absolute values of skin friction drag are about the same in the two gliding positions, it is possible that the increase in this component would be approximately the same. The main difference could occur at the pressure drag since the position with the arms along the trunk presented higher absolute values. It is expected that the drag force decrease in this body position would be more accentuated, thus decreasing the differences between the two models. However, we think these changes would not be sufficient to have an effect on our primary finding: the gliding position with the arms extended at the front produced lower drag coefficients than with the arms placed along the trunk.

Another different situation could happen if the swimmer were at the water's surface. The contribution of the skin friction drag would be reduced owing to the reduction in the wetted area and the generation of wave drag (Bixler et al., 2007).

Although limited to passive drag, this study allowed the evaluation of the effects of different body positions on performance, being a first step toward the analysis of active drag. On the other hand, computational fluid dynamics methods have provided a way to estimate the relative contribution of each drag component to the total drag. Future studies could improve these computational fluid dynamics results by analyzing the passive drag of a swimmer at the water's surface and including wave drag in the measurements. Moreover, the evaluation of the active drag while the swimmer is kicking must also be attempted in the future.

\section{Acknowledgments}

This work was supported by the Portuguese Government by a grant of the Science and Technology Foundation (SFRH/ BD/25241/2005; POCI/DES/58872/2004).

\section{References}

Alves, F., Marinho, D., Leal, L., Rouboa, A., \& Silva, A. (2007). 3-D computational fluid dynamics of the hand and forearm in swimming. Medicine and Science in Sports and Exercise, 39(Suppl. 1), S9.
Bixler, B.S., \& Schloder, M. (1996). Computational fluid dynamics: an analytical tool for the 21 st century swimming scientist. Journal of Swimming Research, 11, 4-22.

Bixler, B., Pease, D., \& Fairhurst, F. (2007). The accuracy of computational fluid dynamics analysis of the passive drag of a male swimmer. Sports Biomechanics, 6, 81-98.

Cossor, J., \& Mason, B. (2001). Swim start performances at the Sydney 2000 Olympic Games. In J. Blackwell, \& R. Sanders (Eds.), Proceedings of Swim Sessions of the XIX Symposium on Biomechanics in Sports (pp. 70-74). San Francisco: University of San Francisco.

Guimarães, A., \& Hay, J. (1985). A mechanical analysis of the grab starting technique in swimming. International Journal of Sports Biomechanics, 1, 25-35.

Jiskoot, J., \& Clarys, J.P. (1975). Body resistance on and under the water surface. In L. Lewillie \& J.P. Clarys (Eds.), Swimming II (pp. 105-109). Baltimore: University Park Press.

Lyttle, A.D., Blanksby, B.A., Elliott, B.C., \& Lloyd, D.G. (1999). Optimal depth for streamlined gliding. In K.L. Keskinen, P.V. Komi, \& A.P. Hollander (Eds.), Biomechanics and Medicine in Swimming VIII (pp. 165-170). Jyvaskyla: Gummerus Printing.

Lyttle, A., Blanksby, B., Elliot, B., \& Lloyd, D. (2000). Net forces during tethered simulation of underwater streamlined gliding and kicking technique of the freestyle turn. Journal of Sports Sciences, 18, 801-807.

Massey, B.S. (1989). Mechanics of Fluids. London: Chapman \& Hall.

Moreira, A., Rouboa, A., Silva, A., Sousa, L., Marinho, D., Alves, F., et al. (2006). Computational analysis of the turbulent flow around a cylinder. Portuguese Journal of Sport Sciences, 6(Suppl. 1), 105.

Polidori, G., Taiar, R., Fohanno, S., Mai, T.H., \& Lodini, A. (2006). Skin-friction drag analysis from the forced convection modeling in simplified underwater swimming. Journal of Biomechanics, 39(13), 2535-2541.

Rouboa, A., Silva, A., Leal, L., Rocha, J., \& Alves, F. (2006). The effect of swimmer's hand/forearm acceleration on propulsive forces generation using computational fluid dynamics. Journal of Biomechanics, 39, 1239-1248.

Rouboa, A., \& Silva, A. (2007). Analysis of the performance parameters of the discus throw. Science \& Sports, 22(1), 14-19.

Silva, A., Rouboa, A., Moreira, A., Reis, V., Alves, F., VilasBoas, J., et al. (2008). Analysis of drafting effects in swimming using computational fluid Dynamics. Journal of Sports, Science, and Medicine, 7(1), 60-66.

Vilas-Boas, J.P., Cruz, M.J., Sousa, F., Conceição, F., \& Carvalho, J.M. (2000). Integrated kinematic and dynamic analysis of two track-start techniques. In R. Sanders, \& Y. Hong (Eds.), Proceedings of the XVIII International Symposium on Biomechanics in Sports, Applied Program - Application of Biomechanical Study in Swimming (pp. 113-117). Hong Kong: The Chinese University Press. 